\title{
Relationships Between Disease Incidence at Two Levels in a Spatial Hierarchy
}

\author{
G. Hughes, N. McRoberts, L. V. Madden, and T. R. Gottwald
}

First author: Institute of Ecology and Resource Management, University of Edinburgh, West Mains Road, Edinburgh EH9 3JG, Scotland UK; second author: Plant Science Department, Scottish Agricultural College, Ayr KA6 5HW, Scotland UK; third author: Department of Plant Pathology, Ohio State University, Ohio Agricultural Research and Development Center, Wooster 44691; and fourth author: USDA Agricultural Research Service, Horticultural Research Laboratory, 2120 Camden Road, Orlando, FL 32803. Accepted for publication 19 February 1997.

\begin{abstract}
Hughes, G., McRoberts, N., Madden, L. V., and Gottwald, T. R. 1997. Relationships between disease incidence at two levels in a spatial hierarchy. Phytopathology 87:542-550.

Relationships between disease incidence measured at two levels in a spatial hierarchy are derived. These relationships are based on the properties of the binomial distribution, the beta-binomial distribution, and an empirical power-law relationship that relates observed variance to theoretical binomial variance of disease incidence. Data sets for demonstrat-

function of incidence at the lower scale, the degree of aggregation at that scale, and the size of the sampling unit. For a random pattern, the relationship between incidence measured at two spatial scales does not depend on any unknown parameters. In that case, an equation for estimating an approximate variance of disease incidence at the lower of the two scales from incidence measurements made at the higher scale is derived for use in the context of sampling. It is further shown that the effect of aggregation of incidence at the lower of the two scales is to reduce the rate of increase of disease incidence at the higher scale.
\end{abstract} ing and testing these relationships are based on observations of the incidence of grape downy mildew, citrus tristeza, and citrus scab. Disease incidence at the higher of the two scales is shown to be an asymptotic
Additional keywords: cluster sampling, Elsinoe fawcettii, Plasmopara viticola, plum pox virus disease.
The choice of spatial and/or temporal scales on which to collect data is among the most important that must be made in ecological research studies (36). This is as true in microbial ecology and plant pathology as elsewhere and underlies attempts to characterize both variability over a range of scales and relationships between different scales $(20,33)$. In pathology, relationships between incidence and severity of disease (32) are perhaps the most familiar examples of data comprising a spatial hierarchy. Drawing on Seem's (32) approach, we formulate some relationships between measurements of disease made at two levels in a spatial hierarchy. Here, however, disease is assessed on an incidence scale at both levels in the hierarchy.

Consider a sampling exercise in which individual leaves are the plant units assessed for the presence or absence of disease. For each plant in the sample, both the total number of leaves and the number of leaves with visual symptoms of disease are recorded. Disease incidence usually is defined as the proportion of plant units diseased (5). Here, the mean proportion of diseased leaves per plant provides an estimate of disease incidence, measured at a spatial scale such that individual leaves are the units of interest. Now, in collecting data on the number of diseased leaves on each plant, plants with at least one leaf diseased (diseased plants) and plants with no diseased leaves (healthy plants) are automatically distinguished, so the proportion of plants diseased can be calculated. Information on disease incidence measured at a spatial scale such that whole plants are the units of interest is available, therefore, without expending any further effort on data collection.

This article is about relationships between such measurements of disease incidence, made at two levels in a spatial hierarchy, and

Corresponding author: G. Hughes; E-mail address: eagr11@castle.ed.ac.uk

Publication no. P-1997-0324-01R

(C) 1997 The American Phytopathological Society the potential application of these relationships in disease assessment and sampling. Different situations in which data on disease incidence at two levels in a spatial hierarchy may arise are considered. These situations are illustrated with data from previously published epidemiological studies $(12-14,25)$ and discussed from the point of view of developing a coherent framework for the analysis and modeling of such data. Relationships between measurements of disease incidence for which the two plant units involved do not comprise successive levels in a spatial hierarchy $(18,23)$ are excluded from consideration.

\section{ASSESSMENTS OF DISEASE INCIDENCE NOT INVOLVING DISEASE PROGRESS}

Theory and data. Madden et al. (25) discussed the incidence of grape downy mildew (caused by Plasmopara viticola) in an experimental vineyard in Ohio. On each of two occasions, in each of three different years, disease incidence was measured by recording the total number of leaves and the number of leaves with visual symptoms of downy mildew. For each of six experimental treatments (comprising different fungicide regimes), disease assessments were made on 15 shoots. As presented here, data for three replicate plots for each treatment have been pooled, thus providing a total of 36 samples (each of 45 shoots) from which the mean proportion of diseased leaves per shoot and the proportion of shoots with at least one diseased leaf both can be calculated. From a sampling perspective, the data were obtained by cluster sampling (17). In this case, the sampling unit ('cluster') was a shoot, and all leaves on each shoot were assessed, on a presence/absence basis, for disease symptoms.

Figure 1 combines the whole of this data set to give an overview of the relationship between these two measurements of incidence. At each assessment, differences in incidence were the result of natural variation in disease and the effects of experimental fun- 
gicide treatments. Variation from treatment to treatment in the total number of leaves was the result of natural variation in leaf number from shoot to shoot. Neither disease progress nor host plant growth are at issue in this case.

In order to provide a quantitative basis for the description of data of this type, a mathematical technique may be used that is already familiar in the context of relationships between disease incidence and disease severity, as discussed by Seem (32). Suppose, for example, that a set of observations of 'number of lesions per leaf' has been collected. The technique relies on the relationship between the zero term of a probability distribution of counts (the probability that a leaf has no lesions, $P[0]$ ) and the mean of the distribution (estimated by the observed mean number of lesions per leaf, $\bar{x}$ ). If the observed data for number of lesions per leaf are fit by the Poisson distribution (often used to describe random counts), $P(0)$ may be estimated by $e^{-\bar{x}}$. Alternatively, if the observed data are fit by the negative binomial distribution (often used to describe aggregated counts), $P(0)$ may be estimated by $(1+\bar{x} / \hat{k})^{-k}$, in which $\hat{k}$ is an estimate of the aggregation parameter of the distribution. In either case, observed mean disease incidence (the proportion of leaves with at least one lesion) is an estimate of $1-P(0)$. Mean disease incidence, therefore, can be written as a function of mean number of lesions per leaf. This methodology has been adopted in a number of different biological contexts, as discussed by Perry $(29,30)$.

In the present context (returning to the grape downy mildew data as an example), a probability distribution that describes the observed frequency of diseased leaves per shoot is first found (by analysis or assumption). 'Diseased leaves per shoot' is a discrete variable that takes values $0,1,2, \ldots$ up to $n$ (where $n$ is the size of the sampling unit; in this example, the total number of leaves per shoot). In this particular case, $n$ varies somewhat, and its value is taken to be the nearest integer to the mean number of leaves per shoot in the data as a whole (thus, here $n=14$ ). A notation is adopted that will be applicable in various situations from which disease incidence data at two levels in a spatial hierarchy arise. The lower of the two scales (in the current example the leaf scale) is denoted by the subscript 'low, while the higher of the two scales (in the current example the shoot scale) is denoted by the subscript 'high.' The mean proportion of diseased leaves per shoot (i.e., observed mean incidence measured at the lower of the two scales) is denoted $\hat{p}_{\text {low }}$. When the size of the sampling unit is constant,

$$
\hat{p}_{\text {low }}=\left(\sum_{i} \hat{p}_{\text {low }, i}\right) / N
$$

$i=1,2, \ldots N ; \hat{p}_{\text {low }, i}$ is the proportion of leaves diseased on the $i$ th shoot; and $N$ is the number of shoots (the shoot is the sampling unit here). Variation in the size of the sampling unit can be accommodated easily in the calculation of $\hat{p}_{\text {low }}$ (25). The proportion of shoots with at least one diseased leaf (i.e., observed mean incidence measured at the higher of the two scales) is denoted $\hat{p}_{\text {high }}$. The proportions $\hat{p}_{\text {low }}$ and $\hat{p}_{\text {high }}$ are estimates of, respectively, $p_{\text {low }}$ (the unobserved probability that a leaf is diseased on a given shoot) and $p_{\text {high }}$ (the unobserved probability that a shoot is diseased).

If the pattern of diseased leaves is random over shoots, the binomial distribution may provide a description of the frequency of diseased leaves per shoot (24). The zero term of the distribution (the probability that a shoot has no diseased leaves, $P[0]$ ) is given by $\left(1-p_{\text {low }}\right)^{n}$. Then:

$$
p_{\text {high }}=1-\left(1-p_{\text {low }}\right)^{n}
$$

The estimate of $p_{\text {high }}$ based on $\hat{p}_{\text {low }}$ is denoted $\tilde{p}_{\text {high }}$ (a tilde is used to denote an estimate that has been made from observations at another spatial scale, rather than directly from observations at the scale denoted by its subscript) and is given by:

$$
\tilde{p}_{\text {high }}=1-\left(1-\hat{p}_{\text {low }}\right)^{n}
$$

Equation $1 \mathrm{~b}$ provides a basis for describing the relationship between disease incidence at two levels in a spatial hierarchy when the pattern of disease is random at the lower of the two scales.

The relationship corresponding to equation $1 \mathrm{~b}$ (with $n=14$ ) is shown as curve (i) in Figure 1. Most of the data points on Figure 1 fall below curve (i), indicating that, in general, at any specified level of disease incidence at the leaf scale, there were fewer diseased shoots than would have been expected if diseased leaves had a random pattern. That is to say, the position of the data points on Figure 1, mostly below curve (i), is indicative of clustering of diseased leaves. Clustered (aggregated) disease incidence data may be described by the beta-binomial distribution $(16,24)$. If the betabinomial distribution is appropriate, $P(0)$ is given by

$$
\prod_{i=0}^{n-1}\left(\frac{1-p_{\text {low }}+i \theta}{1+i \theta}\right)
$$

in which $\Pi(\bullet)$ represents the product function and $\theta$ is the aggregation parameter of the distribution $(\theta \geq 0 ; \theta=0$ corresponds to the binomial; increasing $\theta$ indicates increasing aggregation). Thus, in this case:

$$
p_{\text {high }}=1-\prod_{i=0}^{n-1} \frac{1-p_{l o w}+i \theta}{1+i \theta}
$$

and $\widetilde{p}_{\text {high }}$ is given by:

$$
\tilde{p}_{\text {high }}=1-\prod_{i=0}^{n-1} \frac{1-\hat{p}_{\text {low }}+i \hat{\theta}}{1+i \hat{\theta}}
$$

Equation $2 \mathrm{~b}$ provides a basis for describing the relationship between disease incidence at two levels in a spatial hierarchy when the pattern of disease is aggregated at the lower of the two scales. For the grape downy mildew data partitioned into 36 'groups' (six treatments at each of six disease assessments), an estimate of a common aggregation parameter across groups of $\hat{\theta}=0.133$ (standard error $[$ s.e. $=0.0103$ ) was obtained by maximum likelihood (using the EGRET analysis module PECAN V 0.26.6, Statistics and Epidemiology Research Corporation, Seattle). The relationship corresponding to equation $2 \mathrm{~b}$ (with $n=14$ and $\hat{\theta}=0.133$ ) is shown as curve (ii) in Figure 1.

In the majority of cases, it is probably unrealistic to assume that aggregation (at the lower scale), as characterized by the beta-bino-

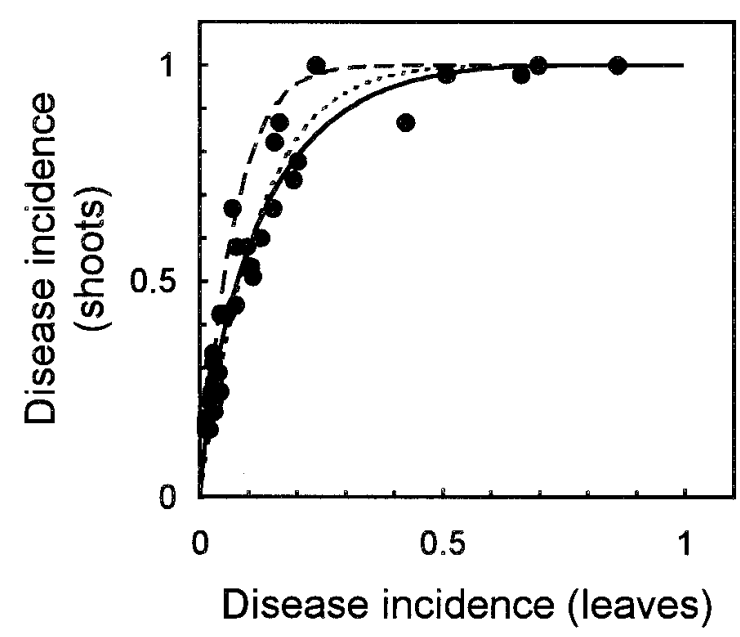

Fig. 1. The relationship between incidence of grape downy mildew on shoots and its incidence on leaves (details of disease assessments are discussed in reference 25). Data derived from disease assessments are indicated by points $(\bullet)$; curve (i) (indicated by the dashed line $[---]$ ) is derived from the binomial distribution; curve (ii) (indicated by the dotted line [-- $]$ ) is derived from the beta-binomial distribution, with $\hat{\theta}=0.133$ (discussed in text); curve (iii) (indicated by the solid line) is derived from the beta-binomial distribution with $\hat{\theta}$ defined as a function of disease incidence at the leaf scale (discussed in text and Fig. 2). 
mial aggregation parameter, does not vary with mean incidence at that scale. However, if there is a linear relationship, on logarithmic axes, between $v_{\text {low }}$ (the variance of the individual observations of disease incidence at the lower of the two spatial scales in the hierarchy), estimated by

$$
\hat{v}_{\text {low }}=\left(\sum_{i}\left[\hat{p}_{\text {low }, i}\right]^{2}-\left[\sum_{i} \hat{p}_{\text {low }, i}\right]^{2} / N\right) /(N-1),
$$

and $v_{\text {low, bin }}$ (the corresponding theoretical binomial variance), estimated by

$$
\hat{p}_{\text {low }} \cdot\left(1-\hat{p}_{\text {low }}\right) / n \text {, }
$$

variation in $\hat{\theta}$ with $\hat{p}_{\text {low }}$ can be described quantitatively (24). Variation in the size of the sampling unit can be accommodated in the calculation of $\hat{v}_{\text {low }}(6,25)$. Madden et al. (25) showed a number of such relationships for the grape downy mildew data used here as an example. Figure 2 shows the relationship for these data grouped as in Figure 1. On this basis, $\hat{v}_{\text {low }}=14.13 \cdot\left(\hat{p}_{\text {low }} \cdot\left[1-\hat{p}_{\text {low }}\right] / n\right)^{1.35}$. Variation in $\hat{\theta}$ then is given by

$$
\hat{\theta}=\frac{\hat{a}-f\left(\hat{p}_{\text {low }}\right) / n}{f\left(\hat{p}_{\text {low }}\right)-\hat{a}},
$$

in which $\hat{a}=\hat{A} \cdot n^{-\hat{b}}$ and $f\left(\hat{p}_{\text {low }}\right)=\left(\hat{p}_{\text {low }} \cdot\left[1-\hat{p}_{\text {low }}\right]\right)^{1-b}$ (with $\hat{A}=14.13$, $\hat{b}=1.35$, and $n=14$ here) (24). With $\hat{\theta}$ so specified as a function of $\hat{p}_{\text {low }}$, use of equation $2 \mathrm{~b}$ results in the relationship shown as curve (iii) in Figure 1.

Fungicide applications affect the temporal dynamics of pathogen populations (10). Thus, in the present case, it can be argued that the fungicide treatments provided 'snapshots' of the spatial dynamics of the grape downy mildew pathosystem, taken from epidemics with different temporal dynamics and at different stages of those epidemics. Nevertheless, a single relationship between observed variance and theoretical binomial variance of disease incidence (illustrated by Fig. 2) serves to summarize spatial pattern of disease incidence at the lower of the two spatial scales. This suggests that the underlying spatial dynamics of the grape downy mildew pathosystem remain similar for a variety of fungicide regimes. It is on this basis that relatively simple models can provide descriptions of the relationship between disease incidence at two levels in a spatial hierarchy. In Figure 1, both of the curves based on equation $2 \mathrm{~b}$ appear, qualitatively, to describe the data rather better than the curve based on equation $1 \mathrm{~b}$. However, none

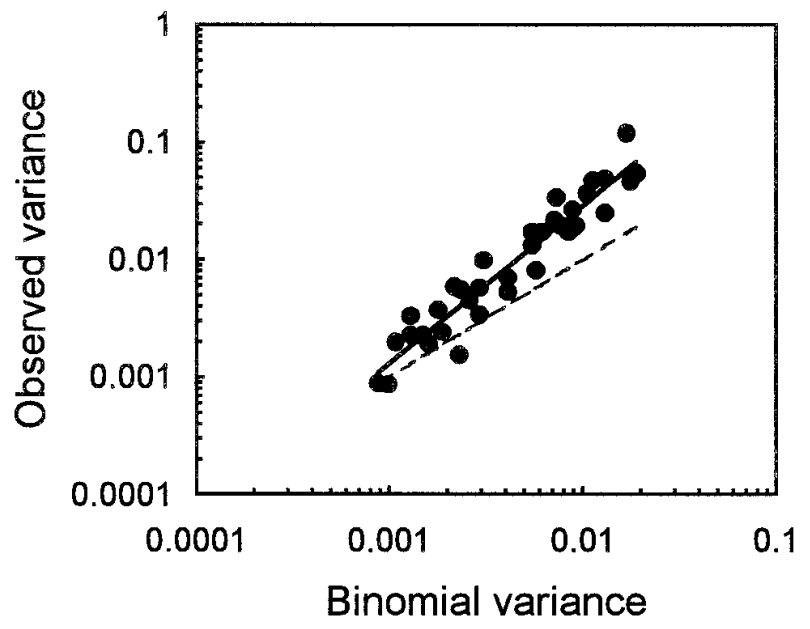

Fig. 2. The relationship between the observed and the theoretical binomial (random) variances of incidence of grape downy mildew at the leaf scale (logarithmic scales are used on both axes). The solid line represents the relationship $\log \left(\hat{v}_{\text {low }}\right)=1.15+1.35 \cdot \log \left(\hat{p}_{\text {low }} \cdot\left[1-\hat{p}_{\text {low }}\right] / n\right)$ fitted to the data by ordinary least squares regression. The dashed line (---) represents the binomial line (i.e., observed variance $=$ binomial variance) . of the curves in Figure 1 have been fitted statistically to the data as shown in the figure. Like the incidence-severity relationships described by Seem (32), the curves are derived solely from distributional analyses of the pattern of disease at the lower of the two spatial scales at which disease has been assessed.

Potential application in disease assessment and sampling. For count data, relationships between mean population density and the proportion of unaffected units have been used as a basis for so-called 'binomial sampling.' This has been adopted more widely in entomology $(1,3,19)$ than in plant pathology (but $[2,8]$ ). Establishment of an incidence-severity relationship allows pathogen population density to be estimated when sampling takes place on a presence/absence basis (hence 'binomial' sampling). Such a procedure may be regarded as advantageous because of the resulting reduction in the resources that need be devoted to sampling.

In order to be able to estimate incidence at the lower scale from incidence at the higher scale, it is possible, in principle, to follow a procedure similar to that on which binomial sampling is based. When disease is assessed on a presence/absence basis at both spatial scales, the term 'binomial sampling' is inappropriate. To emphasize the similarity with binomial sampling, we refer to the use of observations of $\hat{p}_{\text {high }}$ to obtain $\tilde{p}_{\text {low }}$ as 'hierarchical sampling.' In the statistical literature, this type of procedure is referred to as 'group testing.' This has been applied previously in plant pathology and entomology in problems such as the determination of the proportion of vectors carrying a virus from tests of groups of vectors rather than of individuals $(4,34)$ and, more recently, in the assessment of the incidence of virus infection in carnation populations (15).

Equations 1 and 2 provide the basis on which to relate disease incidence at the higher of a pair of spatial scales (which may be less demanding to collect) to incidence at the lower scale. It is straightforward to rearrange equation $1 \mathrm{a}$ :

$$
p_{\text {low }}=1-\left(1-p_{\text {high }}\right)^{1 / n}
$$

Then, mean disease incidence at the lower scale may be estimated from observations made at the higher scale:

$$
\widetilde{p}_{\text {low }}=1-\left(1-\hat{p}_{\text {high }}\right)^{1 / n}
$$

Curve (i) of Figure 3 shows an example of equation $3 \mathrm{~b}$, calculated with $n=4$. Estimates of $\tilde{p}_{\text {low }}$ obtained in this way may be biased (34). The extent of the bias depends on the true value of $p_{\text {low }}$, the number of sampling units $(N)$, and the number of individuals per sampling unit $(n)$. The problem tends to be more serious for small values of $N$ and/or large values of $n$ (34).

An approximate variance for the individual observations of disease incidence at the lower of the two spatial scales in the hierarchy can be calculated from $v_{\text {low }} \approx\left(\mathrm{d} p_{\text {low }} / \mathrm{d} p_{\text {high }}\right)^{2} \cdot v_{\text {high }}$ (7). From equation $3 \mathrm{a}$, the derivative $\mathrm{d} p_{\text {low }} / \mathrm{d} p_{\text {high }}=\left(1-p_{\text {high }}\right)^{(1-n) / n} / n$. This is estimated here by $\mathrm{d} \widetilde{p}_{\text {low }} / \mathrm{d} \hat{p}_{\text {high }}=\left(1-\hat{p}_{\text {high }}\right)^{(1-n) / n} / n$. In the context of cluster sampling (17), the individual observations of disease incidence at the higher of the two spatial scales are either zero or one, since complete sampling units are classified as either 'healthy' or 'diseased.' Thus, $v_{\text {high }}$, the variance of the individual observations at the higher scale, is estimated by $\hat{v}_{\text {high }}=\hat{p}_{\text {high }} \cdot\left(1-\hat{p}_{\text {high }}\right)$. Then,

$$
\tilde{v}_{\text {low }} \approx \frac{\hat{p}_{\text {high }} \cdot\left(1-\hat{p}_{\text {high }}\right)^{(2-n) / n}}{n^{2}}
$$

If required, an approximate standard error for $\tilde{p}_{\text {low }}$ then can be calculated from

$$
\text { s.e. }\left(\widetilde{p}_{\text {low }}\right)=\sqrt{\widetilde{v}_{\text {low }} / N} \text {. }
$$

A numerical example is provided by data from a single assessment of citrus tristeza virus (CTV) disease incidence (T. R. Gottwald, unpublished data). A cluster sampling procedure, with a sampling unit of $n=4$ trees, was used. All 4 trees in each of $N=$ 272 sampling units were assessed, by immunoassay, as either healthy or diseased. The numbers of sampling units with $0,1,2,3$, 
and 4 diseased trees were, respectively, 197, 66, 8, 1, and $0 . \mathrm{Ob}-$ served mean incidence at the lower scale $\left(\hat{p}_{\text {low }}\right)$ was equal to 0.078 . The corresponding variance estimate $\left(\hat{v}_{\text {low }}\right)$ was equal to 0.019 . The observed frequencies are close to the expected binomial frequencies of $196.45,66.59,8.47,0.48$, and 0.01 for $0,1,2,3$, and 4 diseased trees per sampling unit, respectively. Now, suppose that only the presence/absence of disease in sampling units as a whole had been recorded (based, for example, on immunoassay of material taken from the four trees in a sampling unit and pooled) and that the validity of the binomial distribution as a description of the frequency distribution of diseased trees per sampling unit was assumed. A total of 75 out of 272 sampling units were recorded as 'diseased,' so $\hat{p}_{\text {high }}$ is equal to 0.276 . Then, values of $\tilde{p}_{\text {low }}=0.078$ (from equation $3 \mathrm{~b}$ ) and $\tilde{v}_{\text {low }} \approx 0.020$ (from equation 4 ) are obtained, close to the estimates obtained directly from observations at the lower spatial scale.

Equation 2a, for aggregated disease incidence at the lower spatial scale, is more difficult to rearrange than equation 1a. There appears to be no simple, general form. Particular solutions are obtained most easily when $n$ is small and even. For example, when $n=4$ :

$$
p_{\text {low }}=1+\frac{3 \theta}{2}-\frac{1}{2} \cdot \sqrt{5 \theta^{2}+4 \cdot \sqrt{\theta^{4}+(3 \theta+1)(2 \theta+1)(\theta+1)\left(1-p_{\text {high }}\right)}}
$$

Then, mean disease incidence at the lower scale may be estimated from observations of disease incidence made at the higher scale, provided a prior estimate of the aggregation parameter is available:

$$
\widetilde{p}_{\text {low }}=1+\frac{3 \hat{\theta}}{2}-\frac{1}{2} \cdot \sqrt{5(\hat{\theta})^{2}+4 \cdot \sqrt{(\hat{\theta})^{4}+(3 \hat{\theta}+1)(2 \hat{\theta}+1)(\hat{\theta}+1)\left(1-\hat{p}_{\text {high }}\right)}}
$$

The corresponding version of equation $3 b, \widetilde{p}_{\text {low }}=\left(1-\hat{p}_{\text {high }}\right)^{1 / 4}$, is retrieved when $\hat{\theta}=0$. Examples of equation $5 b$ are shown in Figure 3 , curves (ii) and (iii). For curve (ii), $\hat{\theta}=0.5$, whereas for curve (iii), $\hat{\theta}=1.0$ (these values of $\hat{\theta}$ are not estimated from data, but assumed for the sake of illustration). Figure 3 here bears comparison with Seem's Figure 1 (32), showing hypothetical curves representing various shapes for incidence-severity relationships. Variation in $\hat{\theta}$ with $\hat{p}_{\text {low }}$ can be accommodated in curves based on equation $5 \mathrm{~b}$ if a relationship of the form $v_{\text {low }}=A \cdot\left(v_{\text {low }, \text { bin }}\right)^{b}$ has been characterized.

Data from Gottwald et al. (13) on the incidence of plum pox virus disease provide a numerical example of the use of equation 5b. A $6 \times 22$ tree array (reference 13, Fig. 2B) was divided into 33 $2 \times 2$ sampling units ('quadrats'), in one of which there was a missing tree. Ignoring the quadrat with the missing tree, the observed frequencies of $0,1,2,3$, and 4 diseased trees per quadrat were, respectively, 14, 12, 3, 3, and 0 . Observed mean incidence at the lower scale $\left(\hat{p}_{\text {low }}\right)$ was equal to 0.211 . These data were fit by a beta-binomial distribution with $\hat{\theta}=0.116$. The observed frequencies are close to the expected beta-binomial frequencies of $14.30,10.63,5.11,1.66$, and 0.29 for $0,1,2,3$, and 4 diseased trees per quadrat, respectively. Now, suppose that only the presence or absence of disease in quadrats as a whole had been recorded and that the beta-binomial distribution (with $\hat{\theta}=0.116$ ) was assumed to provide a valid description of the frequency distribution of diseased trees per quadrat. A total of 18 out of 32 quadrats were recorded as 'diseased,' so $\hat{p}_{\text {high }}$ is equal to 0.563 . Then, from equation $5 b$ (with $\hat{\theta}=0.116$ ), a value of $\tilde{p}_{\text {low }}=0.216$ is obtained, close to the estimate obtained directly from observations at the lower spatial scale. The corresponding value of $\tilde{p}_{\text {low }}$ obtained from equation $3 \mathrm{~b}$ (based on the binomial distribution) is 0.187 .

When $\tilde{p}_{\text {low }}$ has been obtained from equation $5 \mathrm{~b}$, it is more difficult to obtain $\tilde{v}_{\text {low }}$. For one thing, since equation 5 a is not general, it does not provide a basis for characterizing $\mathrm{d} p_{\text {low }} / \mathrm{d} p_{\text {high }}$ when disease is aggregated at the lower of the two spatial scales. In this case, it is preferable to obtain $\mathrm{d} p_{\text {low }} / \mathrm{d} p_{\text {high }}$ from $\left(\mathrm{d} p_{\text {high }} / \mathrm{d} p_{\text {low }}\right)^{-1}$, since general, albeit rather complicated, forms of $\mathrm{d} p_{\text {high }} / \mathrm{d} p_{\text {low }}$ can be obtained from equation 2 a (discussed in detail in the next section). However, the situation is complicated further by the fact that the aggregation parameter has to be estimated. Sampling variation in $\hat{\theta}$ would need to be taken into account in calculating an approximate value of $\tilde{v}_{\text {low }}$. The effect of sampling variation of estimated parameters on approximate variance estimates has been the subject of extensive discussion in the entomological literature concerning binomial sampling (summarized by Jones [19]). More research on this aspect of hierarchical sampling is required. In particular, the approach outlined by Farrington (9) is worthy of further investigation.

\section{ASSESSMENTS OF DISEASE INCIDENCE INVOLVING DISEASE PROGRESS}

Theory and data. Gottwald et al. (14) discussed the incidence of CTV disease in five orange and grapefruit orchards in Valencia and Alicante provinces, Spain. At each location, annual disease measurements were made over a period in the range of 10 to 13 years. Individual trees were recorded as 'healthy' or 'diseased' by use of monoclonal antibody probes via enzyme-linked immunosorbent assay. For some of the analyses presented by Gottwald et al. (14), the orchard maps were divided into groups of 4 trees arranged $2 \times 2$ ('quadrats'). Viewed in this way, the data arise from a cluster sampling procedure in which the sampling unit was a group of four trees in a rectangular arrangement, and the disease status of each of the four trees in a group was individually assessed. Apart from occasional losses, the number of trees per quadrat remained constant over the period during which data were collected. Both the mean proportion of diseased trees per quadrat (disease incidence at the lower scale) and the proportion of quadrats with at least one diseased tree (disease incidence at the higher scale) were calculated from the data from each disease assessment. Figure 4 shows, separately for each of the five locations, both of these measures of disease incidence changing over time. Differences in disease incidence, resulting from differences in the rate of disease progress, both within and among orchards, are clear.

Figure 5 combines the whole of this data set (a total of 54 disease assessments) to give an overview of the relationship between disease incidence at two levels in a spatial hierarchy. Figure 5 also

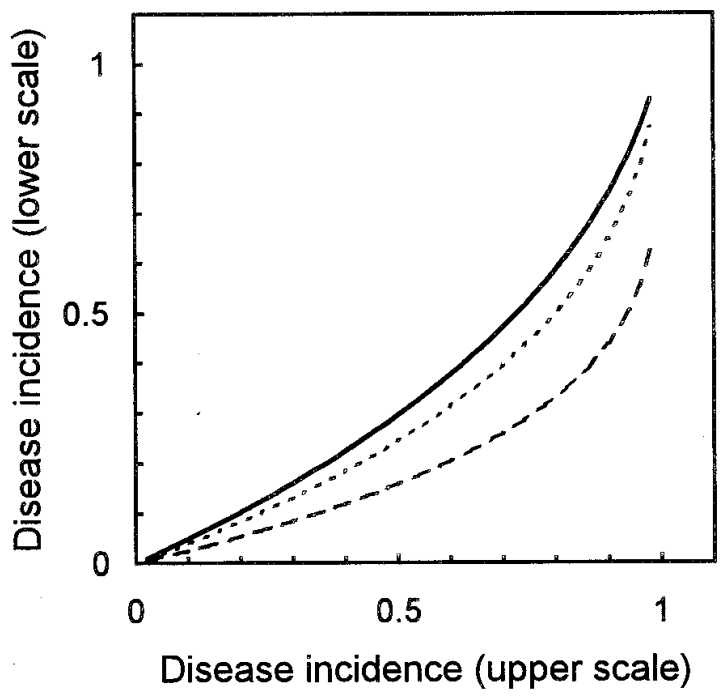

Fig. 3. Curves representing various shapes of relationships between disease incidence at two levels in a spatial hierarchy. Curve (i) (indicated by the dashed line [---]) is derived from the binomial distribution; curve (ii) (indicated by the dotted line [---]) is derived from the beta-binomial distribution with a constant value of $\hat{\theta}=0.5$; curve (iii) (indicated by the solid line) is derived from the beta-binomial distribution with a constant value of $\hat{\theta}=1.0$. 
shows three theoretical relationships, based either on the binomial distribution (equation $1 \mathrm{~b}$, which provides curve [i]) or on the betabinomial distribution (equation $2 \mathrm{~b}$, which provides curves [ii] and [iii]). In each case, $n=4$. Of the two relationships based on the beta-binomial distribution shown in Figure 5, curve (ii) uses an estimate of a common value across disease assessments of $\hat{\theta}=0.0263$ (s.e. $=0.00896$ ), obtained by maximum likelihood (using EGRET, as described previously). Curve (iii) is based on a relationship of the form $v_{\text {low }}=A \cdot\left(v_{\text {low, bin }}\right)^{b}$, shown in Figure 6 (from which the variation in $\hat{\theta}$ with $\hat{p}_{\text {low }}$ can be quantified, as outlined earlier).

Both the closeness to zero of the maximum likelihood estimate of $\hat{\theta}$ and the proximity, in Figure 6, of the regression line through the points to the theoretical line for the binomial distribution indicate that the pattern of diseased trees is close to random. All three curves in Figure 5 are close together and close to the observed data. These curves have not been fitted statistically to the data as shown in the figure. As with Figure 1, the curves are based solely on distributional analyses of the pattern of disease incidence at the lower of the two spatial scales at which disease has been assessed. It is noticeable that while the shapes of the disease progress curves vary considerably among locations (Fig. 4) a single relationship between observed variance and theoretical binomial variance of disease incidence (illustrated by Fig. 6) serves to summarize spatial pattern of disease incidence at the lower of the two spatial scales. Thus, as with the grape downy mildew data (Fig. 1 ), a relatively simple model provides a description of the rela- tionship between disease incidence at two levels in a spatial hierarchy (illustrated by Fig. 5) that serves to summarize the whole of the data set.

Disease progress curves. Waggoner and Rich's (35) discussion of disease progress as influenced by the spatial pattern of propagules provides a theoretical context in which to place the analysis of sequential measurements of disease incidence measured over time ( $t$, arbitrary units) at two levels in a spatial hierarchy. Following Waggoner and Rich (35), we can write:

$$
\begin{aligned}
& \mathrm{d} p_{\text {low }} / \mathrm{d} t=g\left(p_{\text {low }}\right) \\
& \mathrm{d} p_{\text {high }} / \mathrm{d} t=\left(\mathrm{d} p_{\text {low }} / \mathrm{d} t\right) \cdot\left(\mathrm{d} p_{\text {high }} / \mathrm{d} p_{\text {low }}\right)
\end{aligned}
$$

Particular versions of equation 6a require some information or assumption about the forms of $g\left(p_{\text {low }}\right)$ and the derivative $\mathrm{d} p_{\text {high }} / \mathrm{d} p_{\text {low }}$. For logistic increase in disease incidence at the lower scale, $g\left(\hat{p}_{\text {low }}\right)=r_{\text {low }} \cdot \hat{p}_{\text {low }} \cdot\left(1-\hat{p}_{\text {low }} / K_{\text {low }}\right)$. If the binomial distribution describes the pattern of disease incidence at the lower scale, an appropriate formula for $\mathrm{d} p_{h i g h} / \mathrm{d} p_{\text {low }}$ can be obtained from the derivative of equation 1a, estimated in this case by $\mathrm{d} \widetilde{p}_{\text {high }} / \mathrm{d} \hat{p}_{\text {low }}=n \cdot\left(1-\hat{p}_{\text {low }}\right)^{n-1}$. Then:

$$
\begin{aligned}
& \mathrm{d} \hat{p}_{\text {low }} / \mathrm{d} t=r_{\text {low }} \cdot \hat{p}_{\text {low }} \cdot\left(1-\left[\hat{p}_{\text {low }} / K_{\text {low }}\right]\right) \\
& \mathrm{d} \tilde{p}_{\text {ligh }} / \mathrm{d} t=\left(r_{\text {low }} \cdot \hat{p}_{\text {low }} \cdot\left[1-\left\{\hat{p}_{\text {low }} / K_{\text {low }}\right\}\right]\right) \cdot\left(n \cdot\left[1-\hat{p}_{\text {low }}\right]^{n-1}\right)
\end{aligned}
$$

in which $r_{\text {low }}, K_{\text {low }}$, and $n$ are constants: $r_{\text {low }}$ is a rate parameter for disease incidence at the lower spatial scale (units $t^{-1}$ ), $K_{\text {low }}$ is the upper asymptote for disease incidence at the lower scale, and $n$ is
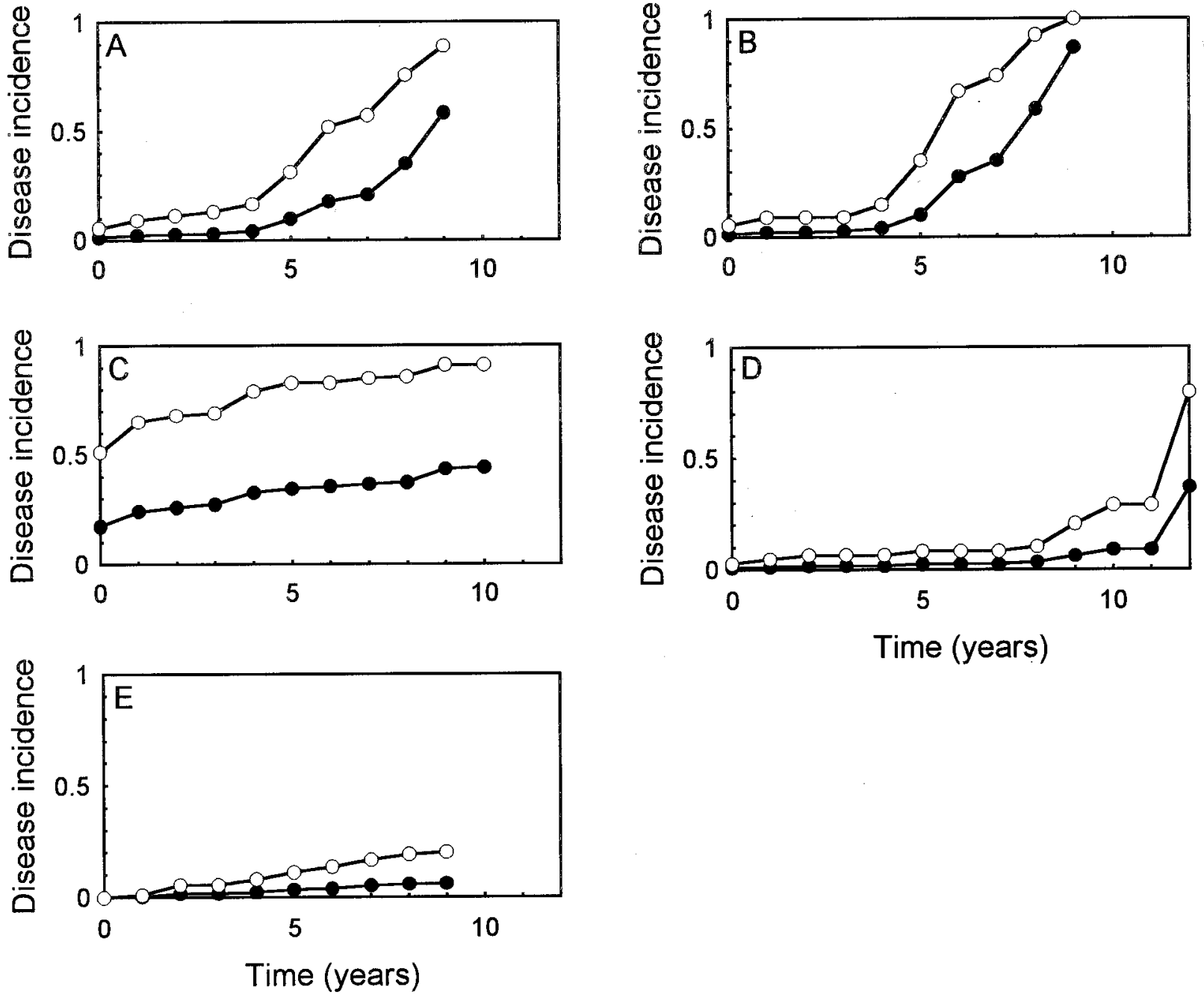

Fig. 4. Temporal progress of citrus tristeza virus disease in five citrus orchards in eastern Spain, shown at two spatial scales (details of disease assessments are discussed in reference 13). Orchards were A, IVIA3\&4; B, IVIV6\&7; C, El Realengo; D, Los Valles; and E, Orihuela. The line joining the solid points (•) represents disease progress when incidence is assessed at the individual tree scale; the line joining the open points (o) represents disease progress when incidence is assessed for sampling units (quadrats) comprising four trees. 
the number of individuals (plants or plant units) per sampling unit. The logistic equation is used here only because of its familiarity and simplicity: another disease progress model could be used instead if it were deemed more appropriate in a particular case. For a numerical illustration, Figure 7A shows the rate of disease progress at the lower scale, $\mathrm{d} \hat{p}_{\text {low }} / \mathrm{d} t$, changing over time, $t$, for equation $6 \mathrm{~b}$ with values of $r_{\text {low }}=0.24$ and $K_{\text {low }}=0.76$. Curve (i) of Figure $7 \mathrm{~B}$ shows the corresponding rate of disease progress at the higher scale, $\mathrm{d} \widetilde{p}_{\text {high }} / \mathrm{d} t$, changing over time, $t$, with $n=9$.

If the beta-binomial distribution describes the pattern of disease incidence at the lower scale, an appropriate formula for $\mathrm{d} p_{\text {high }} / \mathrm{d} p_{\text {low }}$ can be obtained from the derivative of equation $2 \mathrm{a}$, estimated in this case either by:

$$
\frac{\mathrm{d} \widetilde{p}_{\text {ligh }}}{\mathrm{d} \hat{p}_{\text {low }}}=\prod_{i=0}^{n-1}\left(\frac{1-\hat{p}_{\text {low }}+i \hat{\theta}}{1+i \hat{\theta}}\right) \cdot \sum_{i=0}^{n-1}\left(\frac{1}{1-\hat{p}_{\text {low }}+i \hat{\theta}}\right)
$$

or written in terms of gamma functions:

$$
\frac{\mathrm{d} \widetilde{p}_{\text {high }}}{\mathrm{d} \hat{p}_{\text {low }}}=\frac{\Gamma\left(\frac{1}{\hat{\theta}}\right) \cdot \Gamma\left(\frac{1+n \hat{\theta}-\hat{p}_{\text {low }}}{\hat{\theta}}\right) \cdot\left(\psi\left[\frac{1+n \hat{\theta}-\hat{p}_{\text {low }}}{\hat{\theta}}\right]-\psi\left[\frac{1-\hat{p}_{\text {low }}}{\hat{\theta}}\right]\right)}{\hat{\theta} \cdot \Gamma\left(\frac{1+n \hat{\theta}}{\hat{\theta}}\right) \cdot \Gamma\left(\frac{1-\hat{p}_{\text {low }}}{\hat{\theta}}\right)}
$$

in which $\Gamma(\bullet)$ represents the gamma function and $\psi(\bullet)$ represents the digamma function (also called the psi function): if $z$ is a function of $\hat{p}_{\text {low }}, \psi(z)$ represents $\mathrm{d}(\ln [\Gamma\{z\}]) / \mathrm{dz}$. Both the gamma and digamma functions are implemented in Mathcad PLUS 6.0 (Mathsoft, Inc., Cambridge, MA). The effect of aggregation of disease at the lower scale (as characterized by the beta-binomial aggregation parameter) on the rate of disease progress at the higher scale is shown by curves (ii) and (iii) in Figure 7B (both obtained by substituting equation 7a [or 7b] into equation $6 \mathrm{a}$, with $r_{\text {low }}, K_{\text {low }}$, and $n$ as for curve [i]). For curve (ii) in Figure $7 \mathrm{~B}, \hat{\theta}=0.5$, while for curve (iii) $\hat{\theta}=1.0$ (these values of $\hat{\theta}$ are not estimated from data, but assumed for the sake of illustration). Where appropriate, variation in $\hat{\theta}$ with $\hat{p}_{\text {low }}$ can be accommodated in curves based on equation $6 \mathrm{a}$ if a relationship of the form $v_{\text {low }}=A \cdot\left(v_{\text {low }, \text { in }}\right)^{b}$ has been characterized.

Figure $7 \mathrm{C}$ shows a disease progress curve (for the lower spatial scale) that is the integrated version of the curve in Figure 7A. Figure 7D shows three disease progress curves (i, ii, and iii), each being the integrated version of the curve with the corresponding label in Figure 7B. Numerical integration was used in each case.

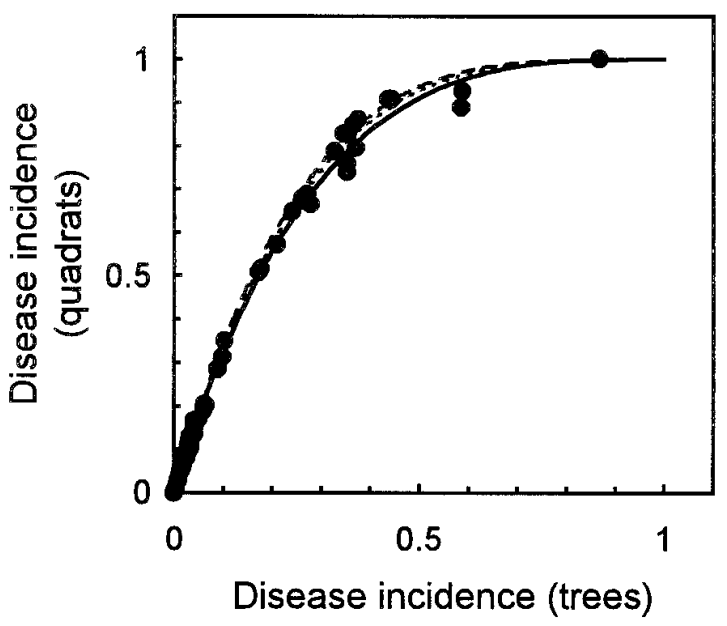

Fig. 5. The relationship between incidence of citrus tristeza virus disease at the quadrat scale and its incidence at the tree scale. Data derived from disease assessments are indicated by points (•); curve (i) (indicated by the dashed line $[---]$ ) is derived from the binomial distribution; curve (ii) (indicated by the dotted line [-- ]) is derived from the beta-binomial distribution with $\hat{\theta}=$ 0.0263 (discussed in text); curve (iii) (indicated by the solid line) is derived from the beta-binomial distribution with $\hat{\theta}$ defined as a function of disease incidence at the tree scale (discussed in text and Fig. 6).
Figure 7 as a whole shows, not surprisingly, that with increasing aggregation of disease incidence at the lower of the two spatial scales, the rate of disease progress at the higher of the two spatial scales is reduced during the early stages of the epidemic. The maximum rate of disease progress at the lower spatial scale occurs when $\hat{p}_{\text {low }}=K_{\text {low }} / 2=0.38$ (and $t=30$ ) (Fig. 7C). At this time, the corresponding values of $\tilde{p}_{\text {high }}$ (Fig. 7D) are $\tilde{p}_{\text {high }}=0.99$ when the pattern of disease incidence at the lower of the two spatial scales is described by the binomial distribution (curve [i]); $\widetilde{p}_{\text {high }}=0.81$ when the pattern of disease incidence at the lower of the two spatial scales is described by the beta-binomial distribution with $\hat{\theta}=0.5$ (curve [ii]); and $\widetilde{p}_{\text {high }}=0.70$ when the pattern of disease incidence at the lower of the two spatial scales is described by the beta-binomial distribution with $\hat{\theta}=1.0$ (curve [iii]) (as above, the values of $\hat{\theta}$ are not estimated from data, but assumed for the sake of illustration). This result corresponds to that of Waggoner and Rich's model (35, equation 8) for the effect of aggregation of pathogen propagules on the rate of disease progress at the plant scale.

\section{ASSESSMENTS OF DISEASE INCIDENCE INVOLVING BOTH DISEASE PROGRESS AND HOST GROWTH}

Theory and data. Suppose that measurements of disease incidence are made at the leaf scale, over a sequence of assessments during which there is an increase both in number of diseased leaves and in the total number of leaves per plant $(n)$. For example, Gottwald (12) discussed the incidence of citrus scab (caused by Elsinoe fawcettii) in four nurseries and eight disease control test plots of sour orange in Florida. Assessments were made of disease progress at the leaf scale and at the tree scale (12, Fig. 1). In this case, the sampling unit was the tree. Incidence at the leaf scale was estimated visually, by trained personnel, due to the impracticability of counting both the total number of leaves and the number showing disease symptoms on each tree.

Figure 8 combines the whole of this data set to give an overview of the relationship between these two measurements of incidence. A simple graphical plot of the disease incidence at the tree scale against disease incidence at the leaf scale appears similar in form to Figures 1 and 5. However, the situation is, from a theoretical point of view, rather more complicated. In equations $1 \mathrm{~b}$ and $2 \mathrm{~b}, n$ was a taken to be a constant for the grape downy mildew and

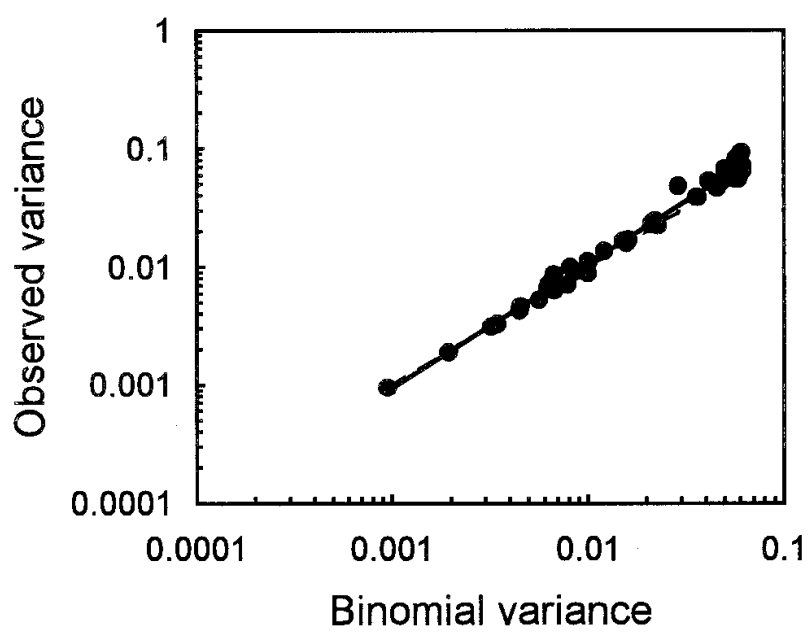

Fig. 6. The relationship between the observed and the theoretical binomial (random) variances of incidence of citrus tristeza virus disease at the tree scale (logarithmic scales are used on both axes). The solid line represents the relationship $\log \left(\hat{v}_{\text {low }}\right)=0.14+1.05 \cdot \log \left(\hat{p}_{\text {low }} \cdot\left[1-\hat{p}_{\text {low }}\right] / n\right)$ fitted to the data by ordinary least squares regression. The dashed line $(---)$ represents the binomial line (i.e., observed variance $=$ binomial variance $)$. 
CTV disease data sets. Now, $n$ (in this case the number of leaves per tree) is a variable, changing over time. In order to show the dependence of disease progress at the higher spatial scale $\left(\widetilde{p}_{\text {high }}\right)$ on two 'independent variables', $\hat{p}_{\text {low }}$ and $n$, a three-dimensional graphical plot would be required.

It is often impractical to count large numbers of leaves, and even when the number of leaves is relatively small, disease assessors, understandably, will be reluctant to count the total number of leaves on plants that are obviously disease-free. Thus, data sets that are sufficiently detailed to allow incorporation of host growth into relationships between disease incidence at two levels in a spatial hierarchy may be encountered relatively infrequently in practice. An alternative scenario for assessments of disease incidence involving disease progress and host growth is outlined in the following section.

Cluster sampling. The main examples used in each of the preceding sections all involved cluster sampling for disease incidence data (17). In each case, incidence was estimated by a form of socalled 'single-stage cluster sampling,' in which every individual leaf on a shoot, tree in a group, or leaf on a tree was taken into account (it is often the case in pathology and entomology that the clusters [sampling units] are arranged systematically rather than randomly, in order to facilitate data collection; discussed below). However, the assessment of every individual in each cluster is not a requirement for cluster sampling. It is possible to subsample from clusters (so-called 'two-stage cluster sampling') to obtain an estimate of (in the present context) disease incidence. Cochran (6) discussed the problem of two-stage cluster sampling for data in the form of proportions and provided a phytopathological example. The most important difference between single- and two-stage cluster sampling is that in the case of the latter, the variance calculation must take into account the variance between the cluster means and, additionally, the variance between individuals within clusters (since only a sample of individuals in each cluster is assessed).

Two-stage cluster sampling represents a valid alternative to the assessment of every individual in a sampling unit when the sampling unit (denoted $n$ in the examples above) corresponding to an obvious natural unit is large. Thus, for example, an estimate of citrus scab incidence could be obtained by assessing a fixed (constant) number of leaves from each tree. The use of a constant subsample size also would avoid the complication of $n$ varying over time (as a result of host growth) when establishing a relationship between disease incidence at two spatial scales.

\section{DISCUSSION}

The relationships outlined in this article are based on a distributional analysis of disease incidence data that is analogous to the
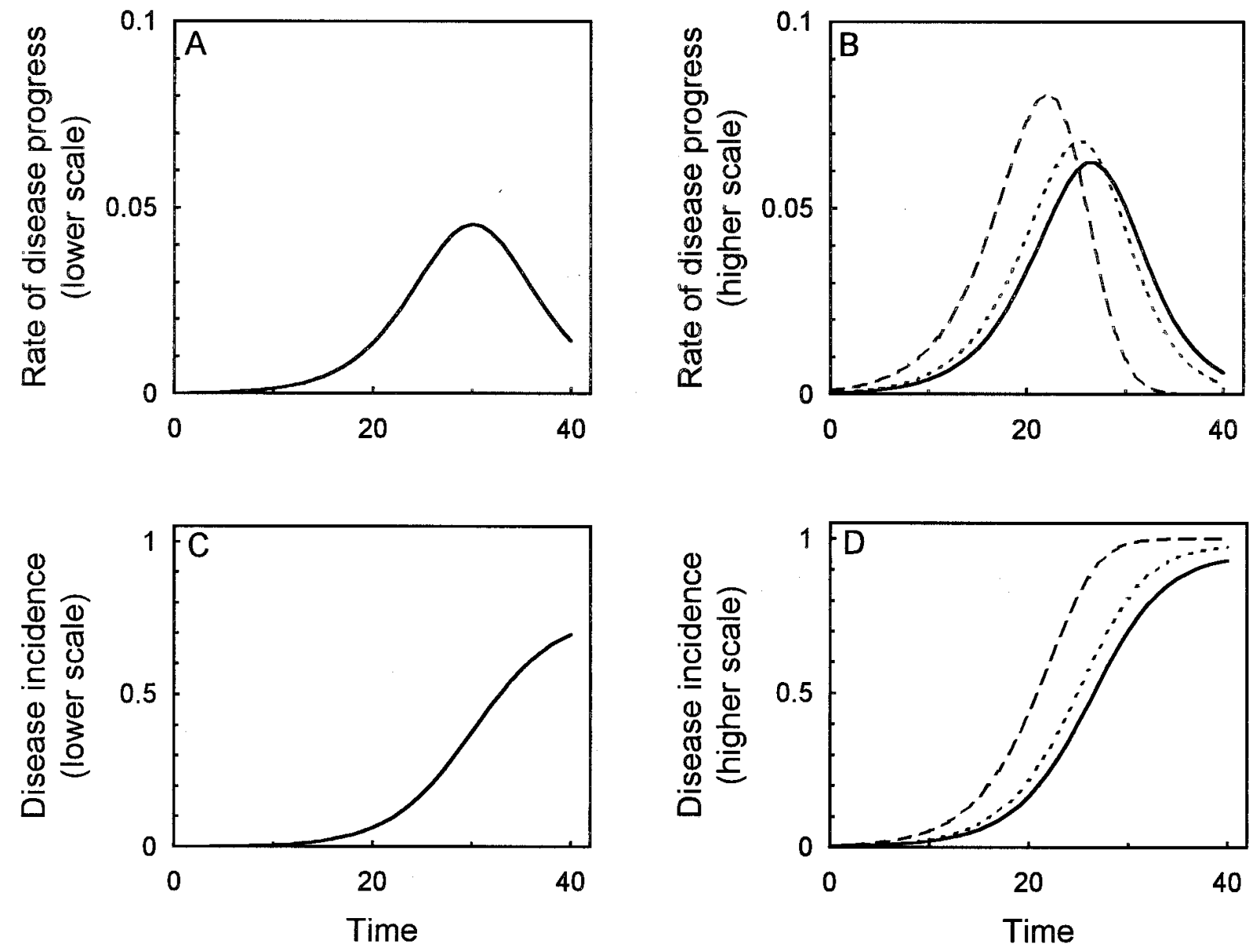

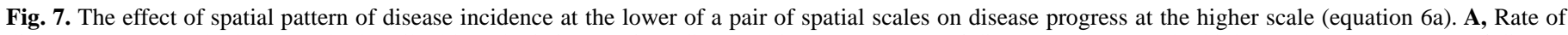

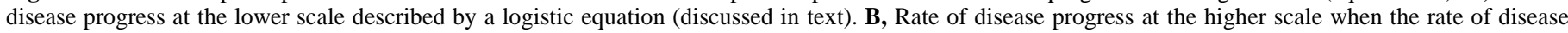

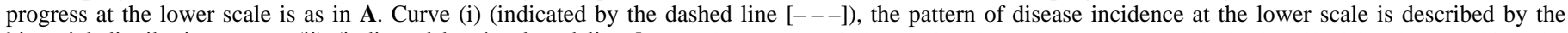

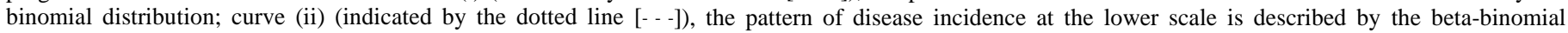

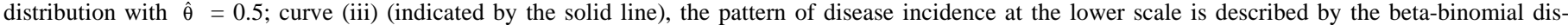

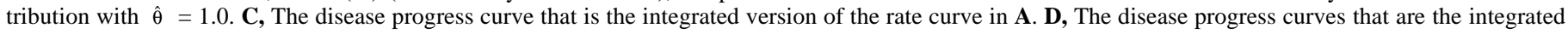

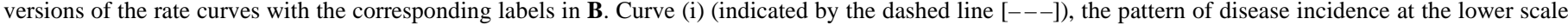

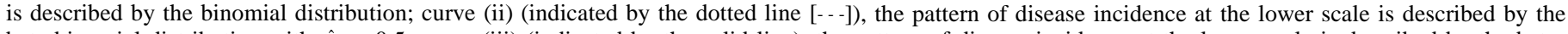

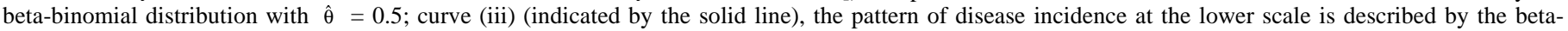
binomial distribution with $\hat{\theta}=1.0$. 
analysis of count data on which Seem (32) based the development of incidence-severity relationships. Assessments of disease incidence at two levels in a spatial hierarchy arise naturally in the context of cluster sampling for disease incidence data. Incidence is assessed both at the (higher) level of the chosen sampling unit and at the (lower) level of the individual members of the sampling unit. When the size of the sampling unit is constant (or nearly so), it is a relatively simple matter to formulate theoretical relationships between the measurements of disease incidence at two levels in a spatial hierarchy, based on the properties of the binomial and beta-binomial distributions. It is noteworthy that the relationship based on the binomial distribution does not require the estimation of any parameter values.

The approach to incidence-severity relationships outlined by Seem (32) requires that 'severity' be a discrete variable, such as the number of lesions per unit area of leaf, taking values $0,1,2, \ldots$ without upper limit. It is, in effect, a measurement of the population density of the pathogen or of disease symptoms. However, most definitions of severity in the phytopathological literature $(5,11,21,27)$ refer to a continuous variable that expresses the amount of diseased plant tissue as a proportion of the total. This is equivalent to the number of lesions per unit area of leaf multiplied by the average area of an individual lesion. As a result, the establishment of incidence-severity relationships for particular pathosystems often relies on empirical curve-fitting $(28,31)$ rather than the distributional approach outlined by Seem (32). Entomologists also use empirical curves as a basis for binomial sampling $(1,19)$. The important point is that the statistical methods used for describing spatial hierarchies should be matched to the type of data that is available (30). This will apply equally if empirical descriptions of relationships between disease incidence at two spatial scales are sought.

Relationships between disease incidence at two levels in a spatial hierarchy can be used to develop methods analogous to binomial sampling for count data. When disease is assessed on an incidence scale at both levels in the spatial hierarchy, we refer to these methods as hierarchical sampling. Of course, the same guidelines that generally apply to cluster sampling for disease incidence $(17,26)$ are still appropriate. For example, preliminary estimates of the mean and variance of disease incidence at the lower spatial scale are required for sample size determination. When the pattern of disease incidence at the lower scale is indistinguishable from random, only a preliminary estimate of mean incidence at that scale is required, since the binomial variance then can be calculated for any specified value of $n$ (the size of the sampling unit). For aggregated patterns of disease incidence at the lower scale, preliminary estimates of both the mean and variance of incidence at that scale are required. The variance depends on the intracluster correlation coefficient, $\rho$. If a preliminary estimate of mean incidence at the lower scale is available and the pattern of incidence at that scale is known to fit the beta-binomial distribution, the intracluster correlation coefficient can be estimated by $\hat{\rho}=\hat{\theta} /(1-\hat{\theta})$. However, aggregation often varies with mean disease incidence. If a relationship of the form $v_{\text {low }}=A \cdot\left(v_{\text {low, bin }}\right)^{b}$ already has been established, the resulting formulae for sample size determination have the advantage of being applicable equally over the whole range of mean disease incidence.

Once the required sample size has been calculated, the positioning of the sampling units in the population to be sampled must be considered. In many types of ecological study, sampling units are selected systematically while traversing a path through a field, violating the assumptions of randomization and independence on which many sampling procedures are based. There is evidence that, in some cases, the effect of these violations appears to be small (22). In the context of crop protection, the effect of these violations depends on the spatial pattern of the particular insect or pathogen population in question (1). In the case of cluster sampling, further investigation of this effect would require an analysis of spatial pattern at the higher scale (i.e., among sampling units). The results of such analyses would certainly enhance our understanding of relationships among patterns at different scales, although whether they would influence the way in which sampling units were selected in the field is moot.

In cluster sampling for disease incidence, sampling units are selected, and incidence is assessed at the scale of the individual members of the sampling unit after classification of each individual in every selected unit as either 'healthy' or 'diseased.' The point of hierarchical sampling is that once the sampling units have been selected, each unit then is classified, as a whole, as either 'healthy' or 'diseased.' Incidence at the lower scale then is estimated from an equation relating incidence at the lower scale to incidence at the higher scale (e.g., equations $3 b$ and 5b). For diseases assessed by visual symptoms, this may reduce time spent on field surveys. For diseases assessed by laboratory assay, fewer resources may need to be devoted to the assay of material collected in the field, since the material from a sampling unit will be combined and assayed as a whole.

As noted by Briolini (3), in the context of binomial sampling, the sensitivity of methods of this kind decreases as the measurement on the higher scale reaches its asymptote. For equations $1 \mathrm{~b}$ and $2 b$, the range of sensitivity of the relationship between incidence at the higher scale and incidence at the lower scale depends on the size of the sampling unit, $n$. With larger values of $n$, the relationship reaches saturation on the higher scale at lower values of incidence at the lower scale. Thus, the smaller the size of the sampling unit (in terms of the number of individuals it contains), the greater the range of sensitivity of the relationship between disease incidence at the higher and lower scales. If the natural sampling unit is large, two-stage cluster sampling should be considered.

The foremost application of relationships between disease incidence at two levels in a spatial hierarchy is likely to be in sampling. The proposed relationships are analogous to relationships between disease incidence and severity, which can be used as a basis for binomial sampling. However, many plant diseases caused by viruses, spiroplasmas, phytoplasmas, and soilborne fungi are assessed only on an incidence scale, and in addition, some modern methods of disease assessment provide incidence data for diseases that previously would have been assessed on a severity scale based on visual symptoms. Such methods may be used in conjunction with hierarchical sampling, provided that the appropriate methods for statistical analysis of disease incidence data from field studies have been applied.

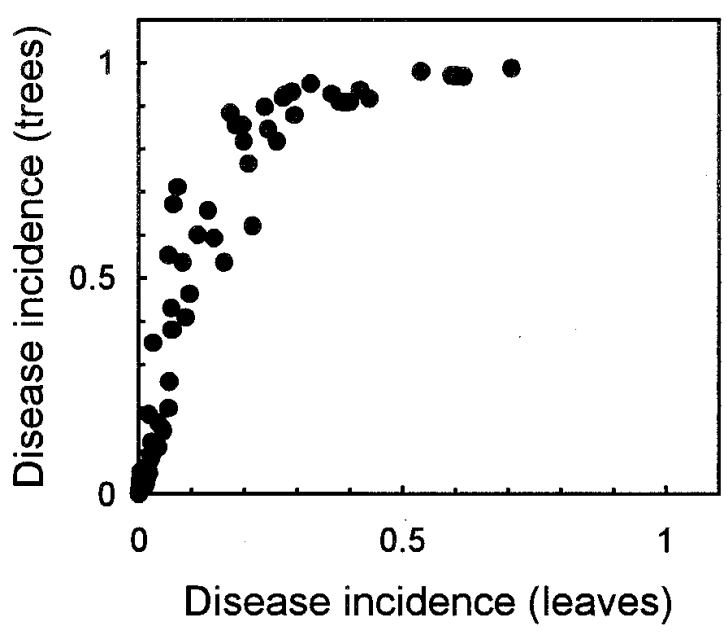

Fig. 8. The relationship between incidence of citrus scab disease at the tree scale and its incidence at the leaf scale (details of disease assessments discussed in reference 11). 


\section{LITERATURE CITED}

1. Binns, M. R., and Nyrop, J. P. 1992. Sampling insect populations for the purpose of IPM decision making. Annu. Rev. Entomol. 37:427-453.

2. Boivin, G., Kushalappa, A. C., and Brodeur, L. 1990. Spatial dispersion and binomial sequential sampling plan for Cercospora carotae on carrots. Can. J. Plant Pathol. 12:209-212.

3. Briolini, G. 1984. Data collection and analysis in pest control. Pages 6774 in: Statistical and Mathematical Methods in Population Dynamics and Pest Control. R. Cavalloro, ed. A. A. Balkema, Rotterdam, Netherlands.

4. Chen, C. L., and Swallow, W. H. 1990. Using group testing to estimate a proportion, and to test the binomial model. Biometrics 46:1035-1046.

5. Chiarappa, L., ed. 1981. Crop Loss Assessment Methods-Supplement 3. Commonwealth Agricultural Bureaux, Slough, UK.

6. Cochran, W. G. 1977. Sampling Techniques. 3rd ed. John Wiley \& Sons, New York.

7. Colquhoun, D. 1971. Lectures on Biostatistics. Clarendon Press, Oxford.

8. de Jong, P. D. 1995. Sampling for detection: Leek rust as an example. Int. J. Pest Manage. 41:31-35.

9. Farrington, C. P. 1992. Estimating prevalence by group testing using generalized linear models. Stat. Med. 11:1591-1597.

10. Fry, W. E. 1982. Principles of Plant Disease Management. Academic Press, New York.

11. Gaunt, R. E. 1987. Measurement of disease and pathogens. Pages 6-18 in: Crop Loss Assessment and Pest Management. P. S. Teng, ed. The American Phytopathological Society, St. Paul, MN.

12. Gottwald, T. R. 1995. Spatio-temporal analysis and isopath dynamics of citrus scab in nursery plots. Phytopathology 85:1082-1092.

13. Gottwald, T. R., Avinent, L., Llácer, G., Hermoso de Mendoza, A., and Cambra, M. 1995. Analysis of the spatial spread of sharka (plum pox virus) in apricot and peach orchards in eastern Spain. Plant Dis. 79:266-278.

14. Gottwald, T. R., Cambra, M., Moreno, P., Camarasa, E., and Piquer, J. 1996. Spatial and temporal analyses of citrus tristeza virus in eastern Spain. Phytopathology 86:45-55.

15. Hepworth, G. 1996. Exact confidence intervals for proportions estimated by group testing. Biometrics 52:1134-1146.

16. Hughes, G., and Madden, L. V. 1993. Using the beta-binomial distribution to describe aggregated patterns of disease incidence. Phytopathology 83:759-763.

17. Hughes, G., Madden, L. V., and Munkvold, G. P. 1996. Cluster sampling for disease incidence data. Phytopathology 86:132-137.

18. Jeger, M. J. 1981. Disease measurement in a study of apple scab epidemics. Ann. Appl. Biol. 99:43-51.
19. Jones, V. P. 1994. Sequential estimation and classification procedures for binomial counts. Pages 175-205 in: Handbook of Sampling Methods for Arthropods in Agriculture. L. P. Pedigo and G. D. Buntin, eds. CRC Press, Boca Raton, FL.

20. Kinkel, L. L., Wilson, M., and Lindow, S. E. 1995. Effect of sampling scale on the assessment of epiphytic bacterial populations. Microb. Ecol. 29:283-297.

21. Kranz, J. 1988. Measuring plant disease. Pages 35-50 in: Experimental Techniques in Plant Disease Epidemiology. J. Kranz and J. Rotem, eds. Springer-Verlag, Berlin.

22. Krebs, C. J. 1989. Ecological Methodology. HarperCollins, New York.

23. Long, D. H., TeBeest, D. O., Rupe, J. C., and Lee F. N. 1996. Distribution of rice blast disease within commercial rice fields in Arkansas. (Abstr.) Phytopathology 86 (Suppl.):S4.

24. Madden, L. V., and Hughes, G. 1995. Plant disease incidence: Distributions, heterogeneity, and temporal analysis. Annu. Rev. Phytopathol. 33: 529-564.

25. Madden, L. V., Hughes, G., and Ellis, M. A. 1995. Spatial heterogeneity of the incidence of grape downy mildew. Phytopathology 85:269-275.

26. Madden, L. V., Hughes, G., and Munkvold, G. P. 1996. Plant disease incidence: Inverse sampling, sequential sampling, and confidence intervals when observed mean incidence is zero. Crop Prot. 15:621-632.

27. Nutter F. W., Jr., Teng, P. S., and Shokes, F. M. 1991. Disease assessment terms and concepts. Plant Dis. 75:1187-1188.

28. Pataky, J. K., and Headrick, J. M. 1988. Relationships between common rust incidence and severity on a susceptible and a partially resistant sweet corn hybrid. Phytopathology 78:1155-1160.

29. Perry, J. N. 1987. Host-parasitoid models of intermediate complexity. Am. Nat. 130:955-957.

30. Perry, J. N. 1994. Sampling and applied statistics for pests and diseases. Aspects Appl. Biol. 37:1-14.

31. Price, T. V., and Williams, B. L. 1990. Studies of the severity and incidence of Papsalum leaf blight. Aust. J. Agric. Res. 41:377-392.

32. Seem, R. C. 1984. Disease incidence and severity relationships. Annu. Rev. Phytopathol. 22:133-150.

33. Shaw, M. W. 1990. A test of spatial randomness on small scales, combining information from mapped locations within several quadrats. Biometrics 46:447-458.

34. Swallow, W. H. 1985. Group testing for estimating infection rates and probabilities of disease transmission. Phytopathology 75:882-889.

35. Waggoner, P. E., and Rich, S. 1981. Lesion distribution, multiple infection, and the logistic increase of plant disease. Proc. Natl. Acad. Sci. USA 78:3292-3295.

36. Wiens, J. A. 1989. Spatial scaling in ecology. Funct. Ecol. 3:385-397. 